\title{
An Exploration of Geographic Routing with $k$-Hop Based Searching in Wireless Sensor Networks
}

\author{
Chung Shue Chen*‡, Yanjun Li ${ }^{\dagger \ddagger}$, Ye-Qiong Song $\ddagger$ \\ ${ }^{*}$ Dept of Electronics \& Telecommunications, Norwegian University of Science and Technology, Norway \\ ${ }^{\dagger}$ State Key Laboratory of Industrial Control Technology, Zhejiang University, China \\ ${ }^{\ddagger}$ LORIA-Nancy University, Viller Les Nancy, France \\ Email: cschen@iet.ntnu.no, yanjun.li@loria.fr, song@loria.fr
}

\begin{abstract}
We explore the asymptotic performance of existing geographic routing with a utilization of $k$-hop neighborhood information. The reachability from source to sink improves as we use more information for routing decision and the average number of hops required decreases significantly from 1-hop to 2-hop searching, which indicates a potential tradeoff between performance enhancement and system complexity. As simple greedy geographic routing is insufficient in lossy wireless environment, we propose a new metric incorporating both advance in distance and link quality. Through simulations, we show its effectiveness over the conventional simple greedy method. The generalization to $k$-hop based routing and resulting performance are also presented. Simulation results show that with the multi-hop based searching, there is a good improvement in the number of transmissions required from source to sink, which also indicates potential improvement in routing delay and energy consumption in transmissions.
\end{abstract}

\section{INTRODUCTION}

Geographic routing was originally developed for packet radio networks in the 1980s [1] but now receives renewed interest as a promising routing candidate for wireless ad hoc and sensor networks due to its attractive properties including stateless nature and low maintenance overhead [2]. In principle, the neighbor node which can forward the packet towards destination in the most progress is selected. Node location is identified through some localization techniques or GPS [3], [4]. It may also involve some information exchange among neighboring nodes. Generally speaking, this greedy method [5] is simple and effective in many cases despite some recovery schemes should be incorporated to handle possible network voids or deadlocks in the delivery [6]. However, it is worth pointing out that most studies in this topic are located on 1-hop neighborhood information based schemes which often address a quite limited optimization. The generalization to 2-hop or more generally $k$-hop based routing has not been profoundly investigated despite interesting and possible [7]. An exploration will be helpful to provide a reference for future design and consideration, especially for the purpose in emerging real-time wireless sensor networks (WSN) [8] and some demanding applications [9].

In the following, we will first conduct a case study of geographic routing in different levels of neighborhood information, says $k$-hop, where $k=1,2,3, \cdots$, and particularly a performance comparison to understand the potential improvement by the extra searching, while assuming the neighborhood information is available. Note that, when $k=\infty$, the result will naturally fall into the classical shortest path routing with global search which is to serve as a benchmark for comparison. In the beginning, a performance study is conducted in an ideal and deterministic WSN model with simple geographic greedy routing determined by the progress in distance. This aims to offer a basic understanding on the performance of $k$-hop based routing while isolated from the impacts of other factors.

In practice, the strategy of simply choosing the neighbor node which is closest to the destination or in greatest distance progress may suffer from low packet reception rate (PRR) [10] or poor link quality with highly variant channel conditions particularly during hops of large distance. This can lead to packet delivery failure and high retransmission cost [11]. A routing metric beyond simple greedy method is thus necessary and favorable. To conduct the study in a lossy and dynamic WSN, we adopt the model used in [12] which also considers link quality transitional regions [10]. Meanwhile, a new routing metric which incorporates the proximity to destination and link reliability is proposed and generalized. Results show that the performance of the new metric outperforms the simple greedy method as expected practically. Furthermore, we investigate the performance in $k$-hop neighborhood based routing under the lossy model and report comparisons.

The rest of the paper is organized as follows. Some related work is discussed in Section II. In Section III, geographic routing in distance metric with $k$-hop searching, where $k=$ $1,2,3$, is studied and compared. The lossy WSN model and new metric are reported in Section IV which includes the corresponding simulation study. Finally, we conclude the paper in Section V.

\section{RELATED WORK}

For system simplicity, most existing routing protocols are based on 1-hop neighborhood information. However, it is promising that multi-hop information can lead to better performance in many aspects including routing, message broadcasting and channel access scheduling [7], [13]-[15]. Details of several distributed information exchange schemes and efficient algorithms in computing 2-hop neighborhood information for wireless ad hoc and sensor networks can be found in [16]-[18]. In a network of $n$ nodes, a complexity analysis is reported in [19] that every node can obtain the knowledge of 2-hop 
neighborhood by a total of $O(n)$ messages, each of $O(\log n)$ bits.

For WSN, there are some geographic distance based routing metrics proposed in the literature. In [7], a link metric called normalized advance (NADV) is proposed for wireless multihop networks. It selects neighbor nodes in tradeoff between link cost and proximity to destination. In general, the link cost can be defined by the application objectives such as delay, energy consumption or link quality. In [12], beyond conventional geographic greedy routing in maximum distance advance which may perform poorly in lossy wireless links, a study of routing strategies in distance-hop tradeoff is conducted. Results show that the product of PRR and the distance traversed towards destination is a routing metric for packet forwarding in general. Our work here is more related to them. The new routing metric proposed in Section IV is inspired by the concept of expected advance per transmission in [7].

It should be noted that, to the best of our knowledge, this paper is the first attempt in exploring geographic routing with $k$-hop neighborhood information for WSN, where generally $k \geq 1$, and giving a comparative study on the asymptotic properties with different levels of hop information. Furthermore, for lossy WSN, new distance-PRR joint metrics are developed in generalization while taking into account multihop information for potential geographic routing schemes.

\section{A Study of Simple Geographic GReEdy Routing IN $k$-HOP NEIGHBORHOOD INFORMATION}

In this section, we extend the conventional geographic greedy routing to the utilization of $k$-hop neighborhood information to investigate the potential improvement when more information is allowed. In principle, the neighbor node which can forward the packet by the most progress is selected as the next hop forwarder. Given $k$-hop neighborhood information, the one in next forwarding which will lead to a node, after $k$ hop routing, closest to the destination is chosen. In generalization, it is chosen by its potential advantage in terms of $k$-hop transmissions identified by the neighborhood information in the corresponding range. The determination follows the same concept of preference in the proximity to destination.

As we know, in geographic routing, a selection of forwarding candidate only by the preference of shortest distance to destination can simply fall into a routing deadlock [13]. In other words, the packet may be passed between two nodes at the dead end. This can be resolved by many different techniques. A simple and effective solution is to blacklist the visited end node and eliminate it from the forwarding candidate list since a loop-back occurs. Here, we follow this approach. Besides, in this Section, the link model adopted is simply a boolean disk, which means if the packet transmission is in the effective radio range, $R$, the delivery is considered successful. A lossy link model will be investigated later in Section IV.

In the performance comparison, the number of hops or transmissions required from source to destination (sink) in the packet forwarding is in our major concern as it reflects both the routing delay and energy consumed during transmissions. Here, we will not take into account MAC and queueing delay in order to focus on the issue of routing. The reachability to be reported soon is defined by the ratio of packets from source which can reach the sink finally. When we find the packet is always buffered at a specific node or possibly looped in an isolated region and thus cannot go into the right direction after a large number of hops, it will be considered as routing failure. This indicator can reflect the routing capability of a path searching scheme. However, significantly, it also depends on the node density and can help to indicate the level of network connectivity.

Nodes in the WSN are uniformly distributed in a geographical area of $(200 \mathrm{~m}, 200 \mathrm{~m})$, while the source and sink are fixed at the location of $(30 \mathrm{~m}, 30 \mathrm{~m})$ and $(170 \mathrm{~m}, 170 \mathrm{~m})$ respectively. Clearly shown in Fig. 1, as the number of nodes increases, the reachability from source to sink increases as well generally due to the resulting better network connectivity. We conduct two sets of simulations with different effective radio range, $R$, of $26 \mathrm{~m}$ and $22 \mathrm{~m}$ respectively, and plot in parallel for reference. As expected, when the number of nodes is insufficient, the reachability will be low even by $\infty$-hop searching due to disjoint clusters or low connectivity between source and sink. It is therefore unsuitable for network formation.

As shown in Fig. 1, when the number of nodes is above 150 in the case $R=26 \mathrm{~m}$, the reachability is at a satisfactory level approximately equal to or above 0.9 . Reasonably, in the case $R=22 \mathrm{~m}$, a higher node density is required to reach this reachability. Detailed studies of critical density for wireless network connectivity can be found in [20], [21]. Here, we will focus on the comparison among different $k$-hop searching results. As observable in Fig. 1, there is an improvement in reachability from 1-hop to 2-hop path searching and also an improvement from 2-hop to 3-hop searching by the fact that some routing difficulties can be earlier identified and better resolved, or even avoided, when more neighborhood and connectivity information is provided. However, generally speaking, the improvement is decreasing as the number of nodes is increasing. For example, the reachability tends to 1 in all the curves when the number of nodes goes to 300 .

It should be noted that the number of hops required from source to sink is an important indicator from the point of view of end-to-end packet delay and energy consumption. This is at the core of our study. As shown in Fig. 2, when the number of nodes increases, the number of hops required decreases as there can be more and better forwarding choices to reach the sink with potentially less hops. An important observation here is that there is a significant improvement in the number of hops required from 1-hop searching to 2-hop searching, while the improvement from 2-hop to 3-hop searching and even more is relatively small. For example, we can clearly see this in the case $R=26 \mathrm{~m}$ and the number of nodes equals to 200 , in which the reachability is almost 1 as known from Fig. 1. Generally speaking, in this system, 2-hop searching is a good option in different $k$ values by its attractive gain in the reduction of required number of hops. Meanwhile, the system 


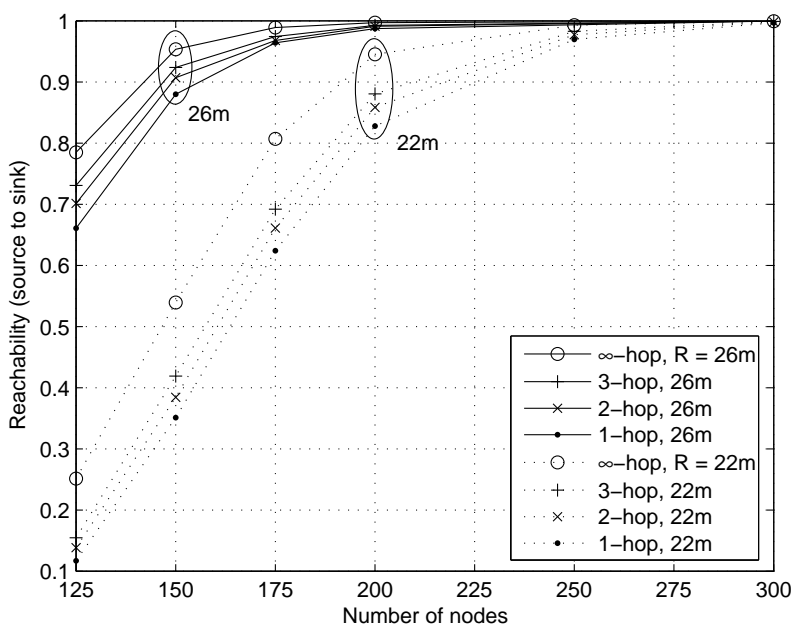

Fig. 1. End-to-end reachability in $k$-hop searching.

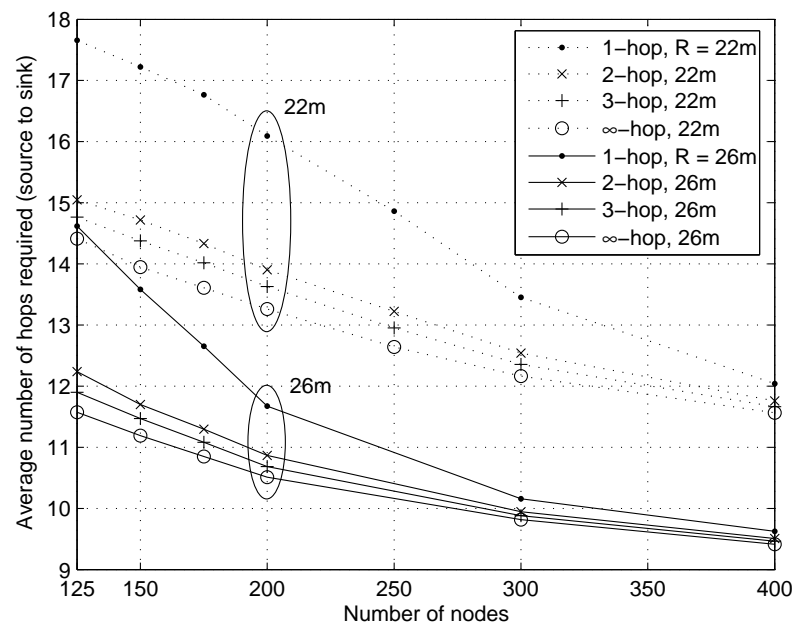

Fig. 2. Average number of hops required in $k$-hop searching

complexity is concerned. Note that, as expected, the gain will get decreased when the number of nodes increases. In addition, by comparing the curves of $R=26 \mathrm{~m}$ and $R=22 \mathrm{~m}$, as the effective radio range is reduced, under same node density, the number of hops required increases generally as in Fig. 2 since the network is now in a lower connectivity. In Fig. 1, the reachability in $R=22 \mathrm{~m}$ drops significantly, especially in the region of relatively low node density, e.g. 125 to 175 .

To have a better understanding on what kind of scenarios the 2-hop searching will take a smaller number of hops than the 1hop searching does, we study the node topology and resulting routing paths by details. Fig. 3 and 4 depict two typical examples observed. As shown in Fig. 3, both searching can find a routing path from source to sink eventually. However, the two searching will go into different paths since node $X$. Node $\mathrm{V}$ is chosen as the next forwarder in the 2-hop searching scheme instead of node $\mathrm{Y}$, although $\mathrm{Y}$ is in fact closer to the

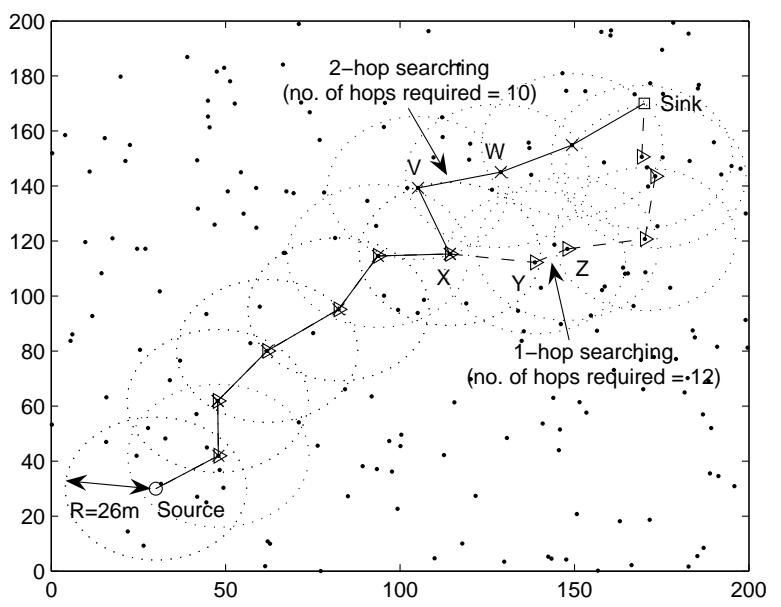

Fig. 3. An example to show the typical scenario in which the 1-hop searching encounters more hops than the 2-hop searching does.

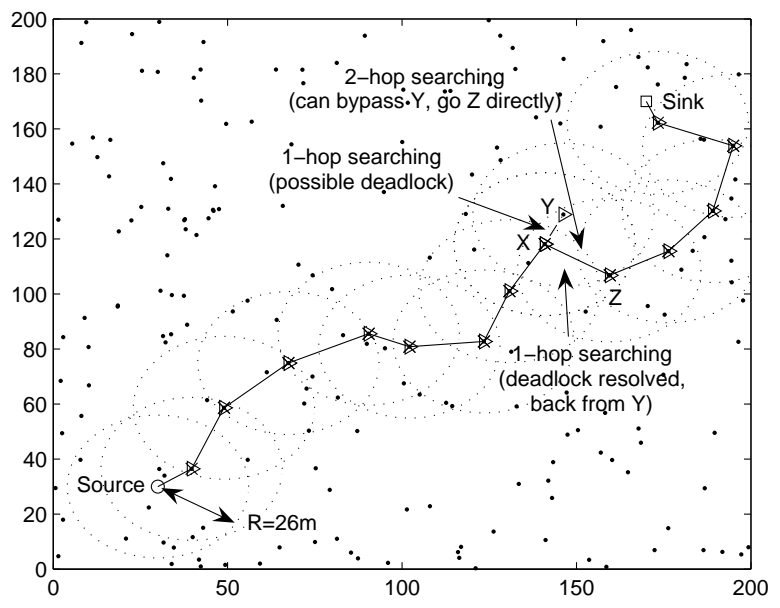

Fig. 4. During the possible deadlock, the 1-hop searching scheme will encounter more transmissions although the deadlock can be resolved.

sink. The reason is the 2-hop searching has identified that in $\mathrm{X}$ 's 2-hop range, via $\mathrm{V}$, node $\mathrm{W}$ is even closer to sink than node $\mathrm{Z}$. So, node $\mathrm{V}$ is better than node $\mathrm{Y}$ from the point of view of 2-hop resulting progress. However, the 1hop searching will simply choose node Y. This effect often helps the 2-hop searching to drive into a shorter path than that of the 1-hop searching.

Fig. 4 shows an example of the 1-hop searching in which a possible routing deadlock occurs and is finally resolved. After forwarded to node $\mathrm{Y}$ from node $\mathrm{X}$, the packet will be routed back to $\mathrm{X}$. As now $\mathrm{Y}$ will be blacklisted, the packet is thus forwarded to node $\mathrm{Z}$ although $\mathrm{Y}$ is at a closer position to sink than Z. However, by the 2-hop searching, when the packet arrives $X$, in the next forwarder selection, the potential progress in 2-hop range will be checked. Clearly, $\mathrm{Y}$ is found as a dead end as it will be back to $\mathrm{X}$. The 2- 
hop searching can thus bypass $\mathrm{Y}$ and forward the packet from $\mathrm{X}$ to $\mathrm{Z}$ directly despite $\mathrm{Y}$ is in fact closer to sink than $\mathrm{Z}$. However, comparatively, the 1-hop searching will encounter more hops (transmissions) and consequently a delay in the deadlock scenario due to the difference in capability. It is worth pointing out that although the 1-hop and 2-hop searching may enjoy close reachability, the number of hops required from source to sink can be quite different generally.

\section{Design of A New Routing Metric And its GENERALIZATION IN LOSSY WSN MODEL}

As reported in [10], a strategy of simply choosing the neighbor node which is closest to sink or in the greatest distance progress may suffer from low PRR or poor link quality simply due to the large distance propagation. This can result in packet delivery failure and high retransmission cost. In this section, we will propose a new routing metric which considers both the distance advance and link quality to be incorporated in the decision at each hop. Motivated by the study and observations in Section III, we generalize the new metric in the scenario of having $k$-hop neighborhood knowledge and compare the performance. The result can help to understand the potential if we can have more of the information for the routing decision in a more practical link model.

\section{A. PRR Model}

Before elaborating on the design, we describe the packet reception rate (PRR) model adopted, which is derived in [10] for lossy WSN links. It is built on experimental measures of practical systems with respect to existing statistical model of time-varying channels. The PRR, $0 \leq p(d) \leq 1$, of a wireless link is expressible as:

$$
p(d)=\left(1-\frac{1}{2} \exp \left(-\frac{\gamma(d)}{2} \frac{1}{0.64}\right)\right)^{8 f}
$$

where $d$ is the transmitter-receiver distance, $\gamma(d)$ is the signalto-noise ratio (SNR), and $f$ is the frame size which equals to 50 bytes including preamble, payload and CRC in following MICA2 motes [22] and the standard non-coherent FSK modulation. This model takes into account both distance-dependent path loss and log-normal shadowing in identifying the signal strength and randomness in wireless environment.

For transmitting power $P_{t}$, the SNR, $\gamma(d)$, is expressible as:

$$
\gamma(d)_{d B}=P_{t d B}-P L(d)_{d B}-P_{n d B}
$$

where, by MICA2 radios, $P_{t d B}$ is set at $0 \mathrm{dBm}$, the noise floor $P_{n d B}$ is at $-115 \mathrm{dBm}$, while the path loss $P L(d)_{d B}$ is modeled as:

$$
P L(d)_{d B}=P L\left(d_{0}\right)_{d B}-10 n \log _{10}\left(d / d_{0}\right)+X_{\sigma d B}
$$

in which $n$ is the path loss exponent, $d_{0}$ is the reference distance ( 1 meter), and $X_{\sigma}$ denotes the log-normal shadowing with zero mean and variance $\sigma^{2}$. In reference to the above model and the discussions in [12], we will adopt the following three settings based on [23]. They represent different environments and characteristics.
1) Sandy flat beach: $n=4.2, \sigma=4.0, P L\left(d_{0}\right)=-40.8$ $\mathrm{dBm}$.

2) Engineering building I: $n=1.9, \sigma=5.7, P L\left(d_{0}\right)=$ $-50.5 \mathrm{dBm}$.

3) Dense bamboo: $n=5.0, \sigma=11.6, P L\left(d_{0}\right)=-38.2$ $\mathrm{dBm}$.

\section{B. Design of Geographic Routing Metric}

As reported in [12], the product of PRR and the distance progress towards destination is a good decision metric. It is often more effective than a purely distance based routing metric in systems with time-varying links due to a better capture of the wireless nature. Instead of simply taking the product of PRR and the distance progress as a metric, we consider the expected proximity to the sink in terms of $p(d)$. The technical details are given below.

Denote the current node by $i$ while its neighbor node by $j$ which also represents the next-hop forwarder candidates. The routing algorithm and simulation for 1-hop based path searching is conducted in the following way:

1) The distance $d_{i, j}$ between pair nodes $i$ and $j$ is assumed known via some localization techniques.

2) The PRR, $p\left(d_{i, j}\right)$, is calculated following (1), (2) and (3). We assume the corresponding PRR statistics are known, i.e. the path loss exponent $n$, log-normal shadowing variance $\sigma^{2}$, and path loss reference $P L\left(d_{0}\right)$.

3) The progress from node $i$ to forwarder candidate $j$ is identified by its expected proximity to destination node, says $D$, defined as:

$$
\bar{d}_{j, D} \triangleq p\left(d_{i, j}\right) \times d_{j, D}+\left(1-p\left(d_{i, j}\right)\right) \times d_{i, D} .
$$

This can also be interpreted as the expected position after 1-hop forwarding despite the fact that a packet cannot be stayed or buffered at that point. However, $\bar{d}_{j, D}$ can serve as the metric in identifying the most suitable candidate.

4) The node with smallest $\bar{d}_{j, D}$ is selected as the next-hop forwarder, denoted as $j^{*}$.

5) The packet is then forwarded to node $j^{*}$. However, this does not mean the transmission is surely successful since the link reliability can be less than 1, i.e. $0 \leq p\left(d_{i, j^{*}}\right) \leq$ 1. There is a success probability of $p\left(d_{i, j^{*}}\right)$ when sending from node $i$ and node $j^{*}$ in the forwarding. In simulation, a packet forwarding will have success probability equal to $p(d)$. When a transmission fails, a retransmission from the current node $i$ will be initiated with new decision making. The decision will be made based on the possibly new network topology, channel variation and link updates. One may also consider it as an ARQ (automatic repeat request) mechanism but with dynamic PRR.

Most geographic routing protocols use 1-hop information, but generalization to 2-hop searching or more is also possible [7]. The above metric uses 1-hop information. A generalization to 2-hop based routing is established below. Here, we assume 
2-hop neighborhood knowledge is obtained via some localization techniques or information exchange. Let's say, the current node is $i$, while its neighbor node is $j$. A neighbor node of $j$ is said $k$. Similarly to (4), by the same concept, we define the following metric to select the best candidate among next-hop forwarders by their expected proximity to sink in 2 hops:

$$
\begin{aligned}
\bar{d}_{j, D}^{(2)} \triangleq & p\left(d_{i, j}\right) p\left(d_{j, k}\right) d_{k, D}+\left(p\left(d_{i, j}\right) \tilde{p}\left(d_{j, k}\right)+\right. \\
& \left.\tilde{p}\left(d_{i, j}\right) p\left(d_{i, j}\right)\right) d_{j, D}+\tilde{p}\left(d_{i, j}\right) \tilde{p}\left(d_{i, j}\right) d_{i, D}
\end{aligned}
$$

where $\tilde{p}(\cdot) \triangleq 1-p(\cdot)$ for the notational convenience. By (5), the neighbor node with smallest $\bar{d}_{j, D}^{(2)}$ is selected as the next-hop forwarder.

Iteratively, a generalization to 3-hop based routing is developed below. Consider the current node is $i$, its neighbor node is $j$, and a neighbor node of $j$ is $k$. The neighbor node of $k$ is said $l$. Similarly to (4) and (5), we select the best candidate in their expected proximity to sink in 3-hop range by the following metric:

$$
\begin{aligned}
\bar{d}_{j, D}^{(3) \triangleq} & p\left(d_{i, j}\right) p\left(d_{j, k}\right) p\left(d_{k, l}\right) d_{l, D}+ \\
& \left(p\left(d_{i, j}\right) p\left(d_{j, k}\right) \tilde{p}\left(d_{k, l}\right)+p\left(d_{i, j}\right) \tilde{p}\left(d_{j, k}\right) p\left(d_{j, k}\right)+\right. \\
& \left.\tilde{p}\left(d_{i, j}\right) p\left(d_{i, j}\right) p\left(d_{j, k}\right)\right) d_{k, D}+ \\
& \left(p\left(d_{i, j}\right)\left(\tilde{p}\left(d_{j, k}\right)\right)^{2}+\tilde{p}\left(d_{i, j}\right) p\left(d_{i, j}\right) \tilde{p}\left(d_{j, k}\right)+\right. \\
& \left.\left(\tilde{p}\left(d_{i, j}\right)\right)^{2} p\left(d_{i, j}\right)\right) d_{j, D}+\left(\tilde{p}\left(d_{i, j}\right)\right)^{3} d_{i, D} \\
= & p\left(d_{i, j}\right) p\left(d_{j, k}\right) p\left(d_{k, l}\right) d_{l, D}+p\left(d_{i, j}\right) p\left(d_{j, k}\right)\left(\tilde{p}\left(d_{i, j}\right)+\right. \\
& \left.\tilde{p}\left(d_{j, k}\right)+\tilde{p}\left(d_{k, l}\right)\right) d_{k, D}+p\left(d_{i, j}\right)\left(\left(\tilde{p}\left(d_{i, j}\right)\right)^{2}+\right. \\
& \left.\tilde{p}\left(d_{i, j}\right) \tilde{p}\left(d_{j, k}\right)+\left(\tilde{p}\left(d_{j, k}\right)\right)^{2}\right) d_{j, D}+\left(\tilde{p}\left(d_{i, j}\right)\right)^{3} d_{i, D} .
\end{aligned}
$$

\section{Simulation Results}

Sensor nodes are uniformly distributed in an area of $(200 \mathrm{~m}$, $200 \mathrm{~m})$, while source and sink are located at $(30 \mathrm{~m}, 30 \mathrm{~m})$ and $(170 \mathrm{~m}, 170 \mathrm{~m})$ respectively. Following (1)-(3), given distance $d$ between a pair of nodes, the corresponding PRR, $p(d)$, is generated in each time instance. The above model and setting refers to a time-varying lossy WSN with dynamic network topology and connectivity. Data are obtained by 3000 runs.

As aforementioned, the following three environment models are employed: (i) sandy flat beach, (ii) engineering building I, and (iii) dense bamboo. Sandy flat beach is considered as common outdoor application environment, while engineering building I refers to indoor situation. Dense bamboo represents a harsh environment. Fig. 5 shows the reachability, while Fig. 6 reports the average number of transmissions required from end to end. Fig. 7 plots the standard deviation of the number of transmissions required in order to indicate the performance stability.

It is observable in Fig. 5(a) that in the lossy WSN, the new routing metric, given by (4), performs a little bit better than the simple (distance only) greedy method in the reachability. However, more importantly, from Fig. 6(a) and 7(a), the number of transmissions required by the new routing metric is significantly less. This is because the simple greedy forwarding considers the proximity to sink only by the physical distance but neglects the reliability in lossy links. Thus, it often tries
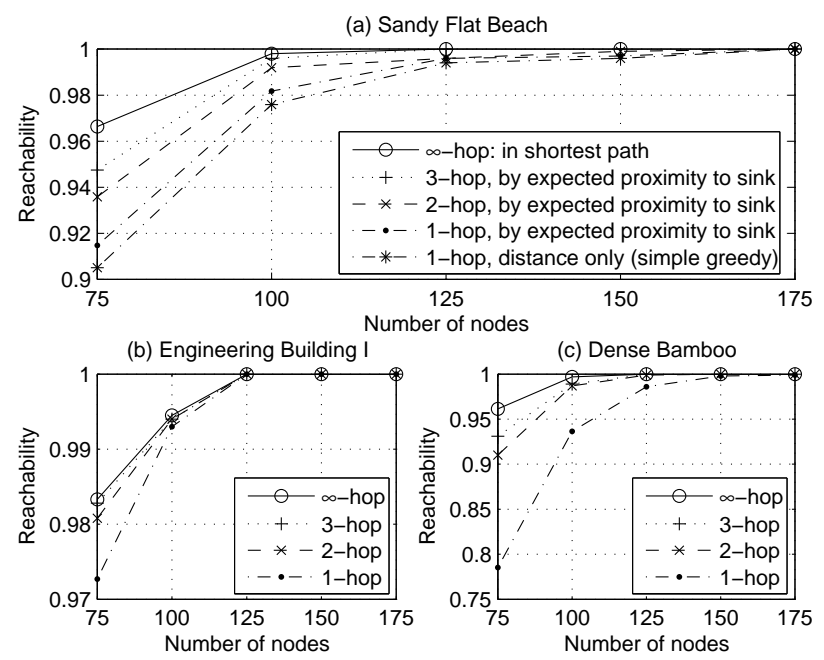

Fig. 5. End-to-end reachability in the three environments.
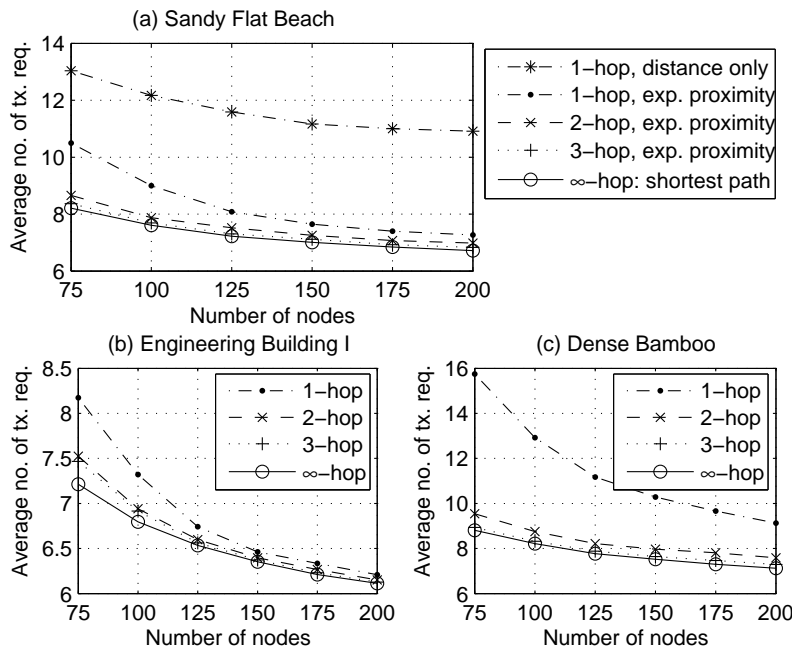

Fig. 6. Average number of transmissions required from source to sink.

(a) Sandy Flat Beach

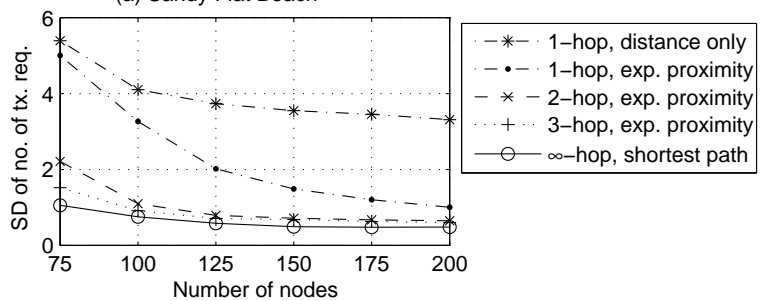

(b) Engineering Building I
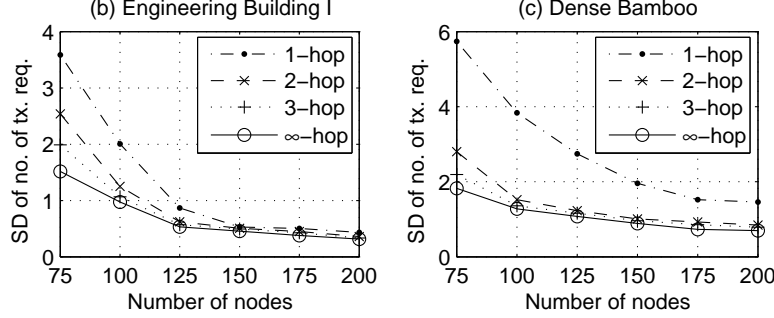

Fig. 7. The standard deviation of the number of transmissions required. 
to propagate in large distance so as to approach sink as much as possible. However, more likely this leads to transmission failure due to corresponding low link reliability, thus results in more retransmissions. On the other hand, the new routing metric has taken into account both the proximity and link quality, and better capture the expected progress per transmission [7]. Discussions on this can also be found in [12].

Regarding the use of multi-hop neighborhood information, there is a clear increase in reachability and decrease in the average number of transmissions required and standard deviation from 1-hop searching to 2-hop searching, under all the three environments, as shown in Fig. 5-7 respectively. The improvement is particularly significant in relatively low node density or in the dense bamboo harsh environment. Results also indicate that in the WSN with high node density and relatively good link quality, e.g. number of nodes around 200 and in engineering building I or sandy flat beach, the gain from 1-hop searching to 2-hop searching is comparatively small. However, in the dense bamboo environment, the improvement is generally substantial.

It is worth pointing out that, look at the number of transmissions required, the improvement from 2-hop searching to 3-hop searching is quite marginal. Besides, as the number of nodes increases, the improvement from a $k$-hop searching to $(k+1)$-hop gets smaller due to the fact that the distance between nodes is now getting shorter. Thus, the PRR will be high between most neighboring nodes. Even if we just adopt the 1-hop searching in the new metric, it could be sufficient to find a good enough routing path. However, without loss of generality, the performance will always be better if more information is allowed with a proper routing strategy.

Comparing results in the three environments, they have shown similar trends. However, in harsh environment of dense bamboo, the magnitude of improvement from 1-hop searching to 2-hop searching is much larger than those in the other two. It indicates the particularly significant advantage in using multi-hop neighborhood knowledge for routing decision in harsh environment or weak links. Generally speaking, the effectiveness in sandy flat beach is still attractive. However, the improvement gets less in the engineering building model.

Note that the $\infty$-hop searching is conducted to find the shortest path and show the least number of transmissions required in terms of packet broadcasting in the lossy WSN. It is to provide a hint of the best performance and just serves as a benchmark for reference. The result refers to an ideal and very optimistic performance.

\section{Conclusions}

The study towards the asymptotic properties of geographic routing with the utilization of $k$-hop neighborhood information gives a general reference of how much improvement we can possibly achieve. The new metric proposed in this paper takes in account of both advance in distance and link quality. It clearly performs better than the simple greedy method. We also study the improvement of the new routing scheme when more information is utilized. It is found that there is a significant improvement in the number of transmissions required from source to sink by the multi-hop information and utilization, especially in harsh environment. It is expected that the results presented here will help to provide a reference for future design and consideration in emerging WSN and certain applications.

\section{REFERENCES}

[1] H. Takagi and L. Kleinrock, "Optimal transmission ranges for randomly distributed packet radio terminals," IEEE Trans. Communication, vol. 32, no. 3, pp. 246-257, March 1984.

[2] I. Stojmenovic, "Position based routing in ad hoc networks," IEEE Commmunications Magazine, vol. 40, no. 7, pp. 128-134, March 2002.

[3] L. Doherty, K. S. J. Pister, and L. E. Ghaoui, "Convex position estimation in wireless sensor networks," in Proc. IEEE INFOCOM, 2001.

[4] T. He, C. Huang, B. Blum, J. Stankovic, and T. Abdelzaher, "Rangefree localization schemes for large scale sensor networks," in Proc. ACM/IEEE MobiCom, 2003.

[5] B. Karp and H. T. Kung, "GPSR: greedy perimeter stateless routing for wireless networks," in Proc. ACM/IEEE MobiCom, 2000, pp. 243-254.

[6] P. Bose, P. Morin, I. Stojmenovic, and J. Urrutia, "Routing with guaranteed delivery in ad hoc wireless networks," Wireless Networks, vol. 7, no. 6, pp. 609-616, 2001.

[7] S. Lee, B. Bhattacharjee, and S. Banerjee, "Efficient geographic routing in multihop wireless networks," in Proc. MobiHoc, 2005, pp. 230-241.

[8] J. Stankovic, T. Abdelzaher, C. Lu, L. Sha, and J. Hou, "Real-time communication and coordination in embedded sensor networks," Proc. IEEE, vol. 91, no. 7, pp. 1002-1022, July 2003.

[9] Y. Li, C. S. Chen, Y. Q. Song, and Z. Wang, "Real-time QoS support in wireless sensor networks: a survey," in Proc. IFAC FET, November 2007, pp. 373-380.

[10] M. Zuniga and B. Krishnamachari, "Analyzing the transitional region in low power wireless links," in Proc. IEEE SECON, 2004, pp. 517-526.

[11] Y. Li, Z. Wang, and Y. Sun, "Analyzing and modeling of the wireless link for sensor networks," Chinese Journal of Sensors and Actuators, vol. 8, pp. 1846-1851, 2007.

[12] K. Seada, M. Zuniga, A. Helmy, and B. Krishnamachari, "Energyefficient forwarding strategies for geographic routing in lossy wireless sensor networks," in Proc. Sensys, 2004, pp. 108-121.

[13] I. Stojmenovic and X. Lin, "Loop-free hybrid single-path/flooding routing algorithm with guranteed delivery for wireless networks," IEEE Trans. Parallel Distrib. Syst., vol. 12, no. 10, pp. 1023-1032, October 2001.

[14] L. Bao and J. J. Garcia-Luna-Aceves, "Channel access scheduling in ad hoc networks with unidirectional links," in Proc. Workshop on Discrete Algorithms and Methods for Mobility, 2001, pp. 9-18.

[15] W. Lou and J. Wu, "On reducing broadcast redundancy in ad hoc wireless networks," IEEE Trans. Mobile Comput., vol. 1, no. 2, pp. 111-122, April-June 2002.

[16] S. Čapkun, M. Hamdi, and J.-P. Hubaux, "GPS-free positioning in mobile ad-hoc networks," Cluster Computing, vol. 5, no. 2, pp. 157167, April 2002.

[17] L. Bao and J. Garcia-Luna-Aceves, "Transmission scheduling in ad hoc networks with directional antennas," in Proc. ACM/IEEE MobiCom, 2002, pp. 48-58.

[18] V. Rajendran, K. Obraczka1, and J. J. Garcia-Luna-Aceves, "Energyefficient collision-free medium access control for wireless sensor networks," Wireless Networks, vol. 12, no. 1, pp. 63-78, Febuary 2006.

[19] G. Calinescu, "Computing 2-hop neighborhoods in ad hoc wireless networks," in Proc. AdHocNow, 2003, pp. 175-186.

[20] P. Gupta and P. R. Kumar, "Critical power for asymptotic connectivity in wireless networks," Stochastic Analysis, Control, Optimization and Applications, pp. 547-566, 1998.

[21] B. Krishnamachari, S. Wicker, R. Bejar, and M. Pearlman, Critical Density Thresholds in Distributed Wireless Networks. Book chapter in Communications, Information and Network Security, 2002.

[22] Crossbow. Mica2 motes datasheet. http://www.xbow.com.

[23] K. Sohrabi, B. Manriquez, and G. J. Pottie, "Near ground wideband channel measurement in 800-1000 MHz," in Proc. IEEE VTC, July 1999 , pp. 571-574. 